\title{
Survivin - biology and potential as a therapeutic target in oncology
}

This article was published in the following Dove Press journal:

OncoTargets and Therapy

15 Octoberber 2013

Number of times this article has been viewed

\author{
Chun Hei Antonio \\ Cheung ${ }^{1,2}$ \\ Chien-Chang Huang ${ }^{3}$ \\ Fang-Ying Tsai ${ }^{4}$ \\ Jane Ying-Chieh Lee' \\ Siao Muk Cheng ${ }^{2}$ \\ Yung-Chieh Chang' \\ Yi-Chun Huang' \\ Shang-Hung Chen ${ }^{5}$ \\ Jang-Yang Chang3,6 \\ 'Department of Pharmacology, \\ College of Medicine, National Cheng \\ Kung University, Tainan, 'Institute \\ of Basic Medical Sciences, College \\ of Medicine, National Cheng Kung \\ University, Tainan, ${ }^{3}$ National Institute \\ of Cancer Research, National \\ Health Research Institutes, Tainan, \\ ${ }^{4}$ South East Asian Health Education \\ Center in Taiwan (SEAHECT), \\ College of Medicine, National Cheng \\ Kung University, Tainan, ${ }^{5}$ Division \\ of Hematology and Oncology, \\ Department of Internal Medicine, \\ Chi-Mei Medical Center, Liouying, \\ Tainan, ${ }^{6}$ Division of Hematology and \\ Oncology, Department of Internal \\ Medicine, National Cheng Kung \\ University Hospital, Tainan, Taiwan, \\ Republic of China
}

Correspondence: Jang-Yang Chang National Institute of Cancer Research, National Health Research Institutes, 2F, No 367 Shengli Road, Tainan 70456, Taiwan, Republic of China

Email jychang@nhri.org.tw
Abstract: Survivin is a member of the inhibitor-of-apoptosis proteins (IAPs) family; its overexpression has been widely demonstrated to occur in various types of cancer. Overexpression of survivin also correlates with tumor progression and induces anticancer drug resistance. Interestingly, recent studies reveal that survivin exhibits multiple pro-mitotic and anti-apoptotic functions; the differential functions of survivin seem to be caused by differential subcellular localization, phosphorylation, and acetylation of this molecule. In this review, the complex expression regulations and post-translational modifications of survivin are discussed. This review also discusses how recent discoveries improve our understanding of survivin biology and also create opportunities for developing differential-functioned survivin-targeted therapy. Databases such as PubMed, Scopus ${ }^{\circledR}$ (Elsevier, New York, NY, USA), and SciFinder ${ }^{\circledR}$ (CAS, Columbus, $\mathrm{OH}$, USA) were used to search for literature in the preparation of this review.

Keywords: survivin, BIRC5, IAP, XIAP, caspase-9, Samc, DIABLO

\section{Introduction}

Survivin (encoded by baculoviral inhibitor of apoptosis repeat-containing 5 [BIRC5]) is a member of the inhibitor-of-apoptosis proteins (IAPs; Figure 1) family found ubiquitously distributed during embryonic and fetal developmental stages. Many studies have shown that survivin, unlike other IAPs, is prominently expressed in the vast majority of neoplasms but not in the differentiated normal tissue. Its overexpression has been demonstrated in breast and lung cancer, prostate, gastric, colon, bladder and esophageal carcinomas, osteosarcomas, and lymphomas. ${ }^{1-4}$ Overexpression of survivin was also found to be significantly associated with a poor prognosis and decreased survival rates in oral, breast and colorectal cancers. ${ }^{5,6}$ In fact, it has been revealed in past studies that patients with survivin overexpressed had a more aggressive and metastatic colorectal carcinoma. ${ }^{6,7}$ In addition, Kawasaki et $\mathrm{al}^{7}$ demonstrated that survivin plays an important role in the transition of colorectal tumorigenesis. Another study done by Hsu et $\mathrm{al}^{8} \mathrm{dem}-$ onstrated that in oral cancer, the extent of survivin expression was negatively correlated with the degree of differentiation. Therefore, survivin overexpression seems to play important roles in the pathogenesis and the progression of various types of cancer.

Because the expression of survivin is positively correlated with tumor progression and inversely correlated with the survival period of the patient after chemotherapeutic treatments, it is important to determine both the molecular regulations and functions of survivin. The survivin gene encodes a $16.5 \mathrm{kDa}$ protein consisting of an $\mathrm{N}$-terminal $\mathrm{Zn}^{2+}$-binding baculovirus inhibitor of apoptosis protein repeat (BIR) domain linked to a 

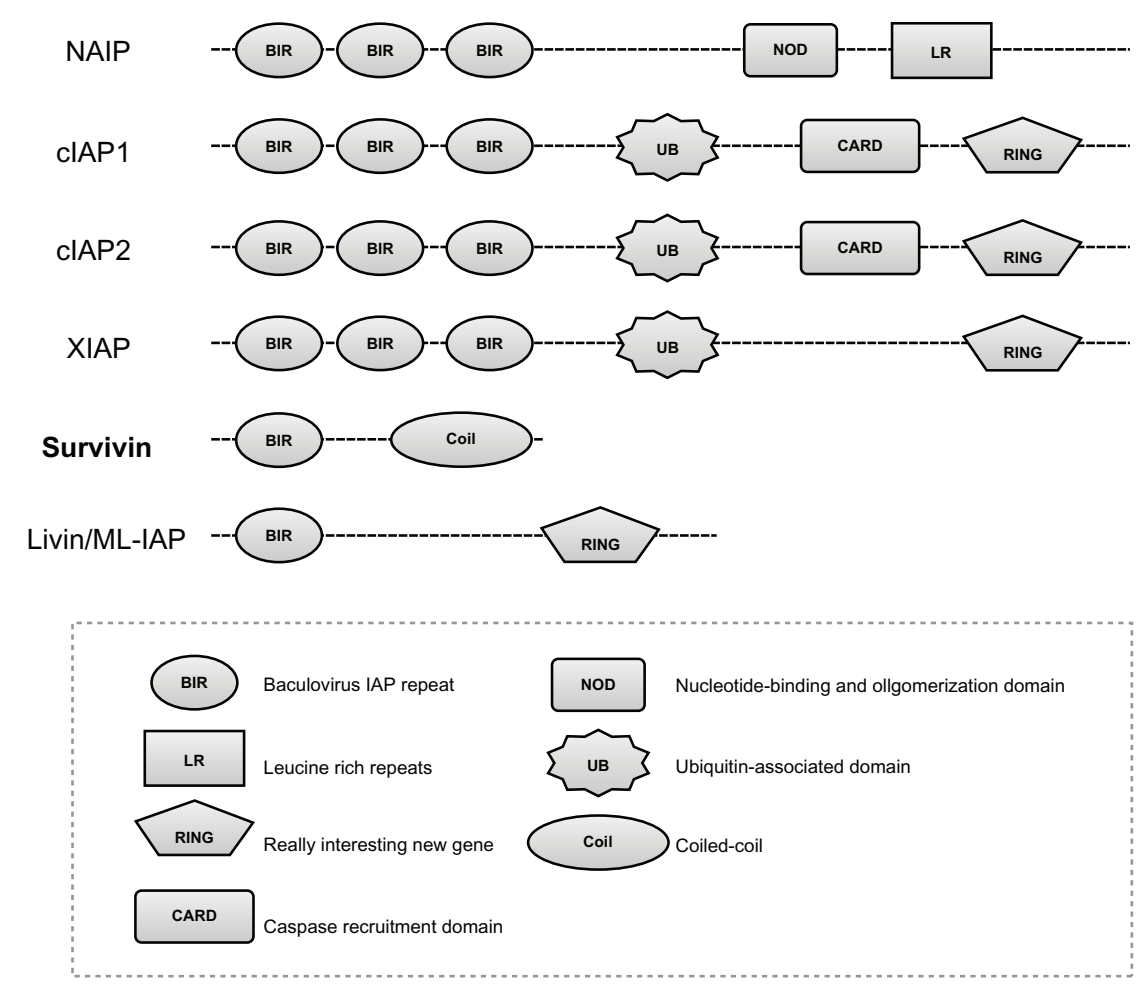

Figure I IAP family members.

Note: Proteins of the inhibitor-of-apoptosis (IAP) family include NAIP, c-IAPI, c-IAP2, XIAP, survivin, and ML-IAP/Livin.

$65 \mathrm{~A}^{\circ}$ amphipathic C-terminal $\alpha$-helix. ${ }^{9}$ Originally, survivin was thought to inhibit caspase-dependent apoptosis through cooperation with the X-linked inhibitor of apoptosis protein (XIAP) and interference with caspase-3/-9. Survivin can also promote mitosis by forming the chromosomal passenger complex (CPC) with aurora-B kinase, inner centromere protein (INCENP), and Borealin. However, recent studies reveal that survivin exhibits multiple anti-apoptotic and pro-mitotic functions, and that the differential functions of survivin seem to be caused by differential subcellular localization, phosphorylation, and acetylation of this molecule. The basic functions of survivin and the current development (both preclinical and clinical) of various survivin inhibitors such as YM155 and SPC3042 for cancer therapy have been discussed in our previously published review. ${ }^{10}$ In this review, we will mainly discuss the complex expression regulations and posttranslational modifications of survivin. We will also discuss how recent discoveries have improved our understanding of survivin biology and also create opportunities for developing differential-functioned, survivin-targeted therapy.

\section{Transcriptional and translational regulation of survivin expression}

Survivin can be regulated at various levels, including transcriptional regulation, post-translational modification, and protein stability regulation. It has been widely demonstrated that deregulation of survivin gene expression is associated with tumorigenesis. Therefore, it is crucial to understand the underlying molecular mechanisms of survivin gene expression and regulation.

Previous studies indicated that regulation of the survivin gene occurs at the level of transcription. ${ }^{11}$ Specificity protein $1(\mathrm{Sp} 1)$ is a human transcription factor involved in gene expression in various cellular development processes. It binds directly onto the DNA promoter region and enhances gene transcription. Given that the survivin promoter lacks a typical TATA or CCAAT box and that Sp1 plays a role in the regulation of genes lacking a functional TATA box, ${ }^{12}$ it is thought that the survivin gene contains several putative $\mathrm{Sp} 1 / \mathrm{Sp} 3$ transcription factor-binding sites. In fact, Xu et al ${ }^{13}$ have revealed that there are two essential binding sites for $\mathrm{Sp} 1$ on the survivin promoter region (one at position -148 to -153 ; the other at position -127 to -140 ) in human $\mathrm{HeLa}$ cervix adenocarcinoma cells, which suggested that $\mathrm{Sp} 1$ is essential in regulating basal survivin promoter activity. In fact, a previous study using electrophoretic gel mobility shift assay (EMSA) showed that $\mathrm{Sp} 3$, a closely related homologue of $\mathrm{Sp} 1$, regulates the survivin promoter in concert with Sp $1 .{ }^{13}$ The same study also revealed that increasing Sp1 or Sp3 levels would induce endogenous survivin expression. 
In contrast, inhibiting $\mathrm{Sp} 1$ or $\mathrm{Sp} 3$ levels by mithramycin or ribonucleic acid interference (RNAi) would decrease the survivin promoter activity. ${ }^{13}$ Interestingly, another study done by Chen et $\mathrm{al}^{14}$ demonstrated that overexpression of Sp1 in A549, a human lung adenocarcinoma cell line, increased survivin expression at both messenger (m)RNA and protein level. This study also revealed that silencing $\mathrm{Sp} 1$ by mithramycin or RNAi decreased survivin expression. Taken together, the Sp1 family serves as an important factor in mediating survivin promoter activity.

The survivin promoter region harbors binding sites for a range of regulatory proteins. Apart from $\mathrm{Sp} 1$ regulation, evidence of $\mathrm{p} 53$ and retinoblastoma (RB)/E2F pathway being involved in survivin gene regulation has also emerged. Using rat embryonic fibroblasts, scientists demonstrated that overexpression and binding of E2F activators (E2F1, E2F2, and E2F3) to the E2F-like binding element increased survivin promoter activity. In contrast, mutation in the E2F DNAbinding domain abolished E2F-induced survivin promoter activity. ${ }^{15}$ Considering the repressive property of RB on E2F activators, Jiang et $\mathrm{a}^{15}$ also demonstrated that RB-inactivation by E1A abrogated the inhibitory effect on survivin transcription in rat embryonic fibroblasts cells. Moreover, the same study revealed that both E2F activators (E2F1 and E2F3) and E2F repressor proteins (E2F4 and E2F5) were able to bind onto the promoter of survivin in human WI-38 embryonic fibroblasts, ${ }^{15}$ suggesting that survivin transcription can be positively regulated by $\mathrm{E} 2 \mathrm{~F}$ activator proteins and negatively regulated by E2F repressor proteins. Another study had also identified an E2F-like binding element spanning from -63 to -53 upstream of the human survivin transcription start site, and both p53 and RB were shown to be essential in repressing survivin transcription in normal human melanocytes. ${ }^{16}$ Because the p53-binding element, located on the -96 to -5 region of survivin promoter, overlaps with an E2F-like binding element, it has been suggested that p53 interferes with the binding between E2F activators and survivin promoter, and subsequently suppresses E2F-mediated survivin gene transcription. Interestingly, recent works on survivin reveal that activation of $\gamma$-secretase induces proteolytic cleavage of Notch, resulting in the release of the Notch-intracellular domain (NICD) and the subsequent translocation of NICD to the nucleus to activate survivin genes transcription. ${ }^{17,18}$

Evidence also points to non-transcriptional mechanisms controlling survivin levels in tumor cells. Vaira et a ${ }^{19}$ demonstrated that insulin-like growth factor-1 (IGF-1) up-regulated survivin expression by promoting translation of survivin in the human DU145 prostate cancer cell line. Since previous studies had shown that IGF-1 promoted cellular proliferation through activating phosphoinositide 3-kinase (PI3K)/protein kinase B (AKT)/mammalian target of rapamycin (mTOR) signaling pathway, ${ }^{20}$ Vaira et al treated prostate cancer cells with rapamycin, an mTOR inhibitor, to investigate whether the mTOR pathway was involved in the regulation of IGF1 -induced survivin expression. Their data showed a novel survival circuitry initiated by IGF-1/AKT signaling, and involving $\mathrm{mTOR}$ activation, $\mathrm{p} 70 \mathrm{~S} 6 \mathrm{~K}$ activation and increased translation of the survivin mRNA.

\section{Regulations of survivin protein stability Cell-cycle dependent regulation of survivin}

In addition to regulating survivin expression at the transcriptional and translational levels, its expression can also be regulated through altering protein stability. Survivin is shown to be clearly regulated by the cell cycle, as its expression is found to be dominant in the G2/M phase. ${ }^{21}$ Interestingly, some studies show that the up-regulation of survivin in the $\mathrm{G} 2 / \mathrm{M}$ phase is mainly caused by reduced rate of protein degradation instead of increased rate of gene transcription in cells. ${ }^{22}$ It was demonstrated in a study done by Zhao et $\mathrm{al}^{22}$ that the level of endogenous survivin was increased in human embryonal kidney 293 cells arrested in the G2/M phase. In contrast, the level of endogenous survivin was shown to be greatly reduced in 293 cells arrested in the G1 phase. Furthermore, the reduction in survivin levels during G1 phase was abolished by the application of proteasome inhibitors, MG132 and lactacystin. ${ }^{22}$ On the basis of these findings, cell cycle dependent pattern of survivin expression and degradation was proposed to be associated with the ubiquitin-proteasome pathway.

\section{Heat shock protein 90 mediated survivin protein stability}

Cells express several molecular chaperones to ensure the correct folding of proteins upon protein synthesis. The chaperones are also required to refold proteins that can become denatured when cells are exposed to stresses such as abnormally high temperatures. Heat shock protein 70 (Hsp70) and heat shock protein 90 (Hsp90) are the most important molecular chaperones in mammalian cells. Unlike Hsp70, Hsp90 does not serve to fold nascent proteins. Rather, Hsp90 binds to substrate proteins that are in a near-native state and thus at a late stage of folding. ${ }^{23}$ Some scientists have suggested that 
the adenosine triphosphate (ATP)-bound state of Hsp90 binds stably to substrate polypeptides that are held by an internally dimerized clamp, and substrate release is achieved through the process of ATP hydrolysis and associated conformational changes within Hsp90. ${ }^{23}$ Survivin associates with Hsp90 in vitro via an interaction that involves the ATPase domain of Hsp90. Evidence of direct binding between Hsp90 and survivin is supported by an enzyme-linked immunosorbent assay (ELISA)-based receptor-ligand assay. ${ }^{24}$ The expression of both Hsp90 and survivin in HeLa cells increased in a time-dependent manner after heat shocking the cells. In contrast, treatment of HeLa cells with an Hsp90 inhibitor geldanamycin resulted in a concentration-dependent loss of survivin expression. Importantly, the loss of survivin after Hsp90 inhibition was also shown to be caused by proteasomal-dependent destruction. ${ }^{24}$

\section{Growth factor-induced survivin protein stability}

So far, we have discussed the effect of Sp1/Sp3, RB/E2F, mTOR, and Hsp90 on the regulation of survivin expression. However, that is not the end of the story. Recent studies reveal that the proteasomal degradation of survivin can also be affected by activation of various growth factor signaling pathways. For example, a study carried out by Wang et $\mathrm{al}^{25}$ showed that treatment with nutrients and growth factors, including glucose and epidermal growth factor (EGF), prompted an increased survivin protein expression in both mouse MIN6 and rat INS-1 pancreatic $\beta$-cells without significant increase in survivin mRNA levels or activation of survivin promoter. Moreover, the addition of EGF was shown to prevent polyubiquitination of survivin, thereby protecting survivin from degradation. ${ }^{25}$ Studies done by $\mathrm{Ju}$ et $\mathrm{al}^{26}$ and Siddiqa et $\mathrm{al}^{27}$ also consistently unmasked the relationship between the stability of survivin and EGF receptor (EGFR) signaling pathway in human breast cancer cells. Both studies revealed that survivin expression was up-regulated via extracellular signal-regulated kinases (ERK) activation and PI3K signaling in EGFR2 (HER2) activated human breast cancer cells through altering the protein stability of survivin. As we have discussed in previous sections, in response to cell death stimulation, survivin physically associates with XIAP; this complex in turn promotes enhanced XIAP stability and synergistic inhibition of caspase- 9 activation. In contrast, the XIAP-associated factor 1 (XAF1) is a putative tumor suppressor that antagonizes the anti-apoptotic molecule XIAP. XAF1 binds to XIAP and subsequently activates XIAP's ubiquitin-protein isopeptide ligase (E3) activity to catalyze survivin ubiquitination. Activation of HER2/ERK was shown to reduce the formation of the XIAP-XAF1 complex, which catalyses ubiquitin-dependent degradation of survivin, leading to an increase in survivin protein stability. ${ }^{26}$ The regulation of survivin protein stability by HER2 is even more complicated: HER2 not only activates the ERK signaling pathway, but it also activates PI3K/AKT pathway. ${ }^{26}$ The HER2/AKT pathway prompts a phosphorylation cascade that increased cyclin-dependent kinase 1 (CDK1/p34 $\left.{ }^{\text {cdc2}}\right)$-cyclin B1 phosphorylation on threonine 161 (Thr161) and subsequently increased the phosphorylation of survivin on $\mathrm{Thr} 34 .{ }^{26}$ As reported in previous studies (and as will be discussed in the following sections), phosphorylation on Thr34 is required to maintain the stability of the protein. ${ }^{28}$ Thus, HER2/ERK and HER2/AKT signalling pathways act in concert to enhance the stability of survivin. Some scientists have also suggested that in addition to EGF, survivin expression can also be regulated by the vascular endothelial growth factor (VEGF) and the platelet-derived growth factor (PDGF). ${ }^{29-31}$ The results of a study by Beierle et $\mathrm{al}^{29}$ showed a consistent finding where exposure to VEGF induced PI3K/AKT pathway activation, which resulted in an increased survivin protein expression in neuroblastoma cells.

\section{Post-translational modifications of survivin Phosphorylation-diversified activities of survivin}

It has been widely demonstrated that survivin is overexpressed in nearly all kinds of cancer cells, with minimal expression in the differentiated normal tissues. In addition, survivin plays important roles in both the process of mitosis and the inhibition of apoptosis. Although it has long been known that mutation in the promoter region of survivin and deregulation of its related transcription factors may lead to the overexpression of survivin in cancer cells, ${ }^{32-34}$ the mechanisms underlying the transition of survivin functions were largely unknown in the past. Recent works on survivin reveal that post-translational modification including phosphorylation and acetylation plays an important role in regulating cellular function of survivin. In fact, survivin can be phosphorylated at multiple sites (eg, threonine 34, 53, and 117; serine 20) and that these modifications can drive survivin to exhibit different molecular functions.

Phosphorylation of survivin on Thr34 by CDK1/p34 ${ }^{\text {cdc2 }}$ cyclin B1 was observed in the human cervix adenocarci- 
noma cells, oral submucous fibrosis and oral squamous cell carcinoma cells. ${ }^{35-37}$ Unlike other members of the IAPs, survivin only contains a single IAP-specific BIR domain, and the amino acid residue Thr34 lies within this domain. O'Connor et $\mathrm{al}^{35}$ showed that Thr34 phosphorylated survivin bound to caspase-9 in HeLa cells. In contrast, Thr34 phosphorylation defective survivin was shown to be incapable of forming a complex with caspase-9. Furthermore, a phosphorylated dead mutation Thr34 $\rightarrow$ alanine (T34A) survivin was developed in the same study, where the researchers demonstrated that the T34A survivin mutant could induce caspase 9-dependent apoptosis in HeLa cells. ${ }^{35}$ Therefore, it is suggested that survivin phosphorylation on $\mathrm{Thr} 34$ by $\mathrm{p} 34^{\mathrm{cdc} 2}$ may regulate intrinsic apoptosis (Figure 2). In fact, a recent study also shows that phosphorylation of survivin at Thr34 enhances its cytoprotective effect in cancer cells. ${ }^{38}$

On the other hand, phosphorylation of survivin on serine 20 (Ser20) and threonine 117 (Thr117) displays distinct functions comparable to the phosphorylation mentioned previously. Two studies have indicated that protein kinase A (PKA) and polo-like kinase 1 (PLK1) catalyze the phosphorylation of survivin on Ser20. ${ }^{39,40} \mathrm{PKA}$ is a serine/threonine kinase; its activity is associated with increased cell survival. Scientists have shown that PKA phosphorylates survivin on Ser20 in the cytosol, but not in mitochondria. In mammalian cells, survivin is located in both the mitochondria and the cytosol, and mitochondrial survivin seems to play an important role in inhibiting the process of apoptosis. ${ }^{41}$ It is believed that in response to pro-apoptotic stimulation, mitochondrial Ser20dephosphorylated survivin is rapidly released into the cytosol, where it prevents caspase activation and inhibits apoptosis through physical interactions with XIAP. A study done by Dohi et $\mathrm{al}^{39}$ demonstrated that PKA-mediated Ser20 phosphorylation interfered with the binding capacity between survivin and XIAP in the cytosol of INS-1 cells. In addition, transfection of non-PKA phosphorylatable survivin was shown to be capable of enhancing XIAP stability and subsequently inhibit apoptosis. ${ }^{39}$ Therefore, phosphorylation of survivin at Ser20 decreases its anti-apoptotic activity.

Interestingly, $\mathrm{Chu}$ et $\mathrm{al}^{40}$ have illustrated that survivin Ser20 is also one of the substrates of PLK1. PLK1 is a multitasking protein that can regulate mitotic entry, centrosome separation, spindle assembly, and chromosome alignment. Besides PLK1, the chromosomal passenger complex (CPC) is also a key regulator of mitosis; this complex is composed of survivin, Borealin, INCENP, and Aurora B kinase. ${ }^{42,43}$ In the study carried out by $\mathrm{Chu}$ et al, ${ }^{40}$ PLK1-mediated phosphorylation of survivin was shown to catalyze Aurora $\mathrm{B}$ activation. Subsequently, Aurora B attached to spindle microtubule and formed a $\mathrm{CPC}$, which plays an important role in cytokinesis. ${ }^{40,44}$ Taken together, phosphorylation of survivin on Ser20 seems to regulate the differential functions

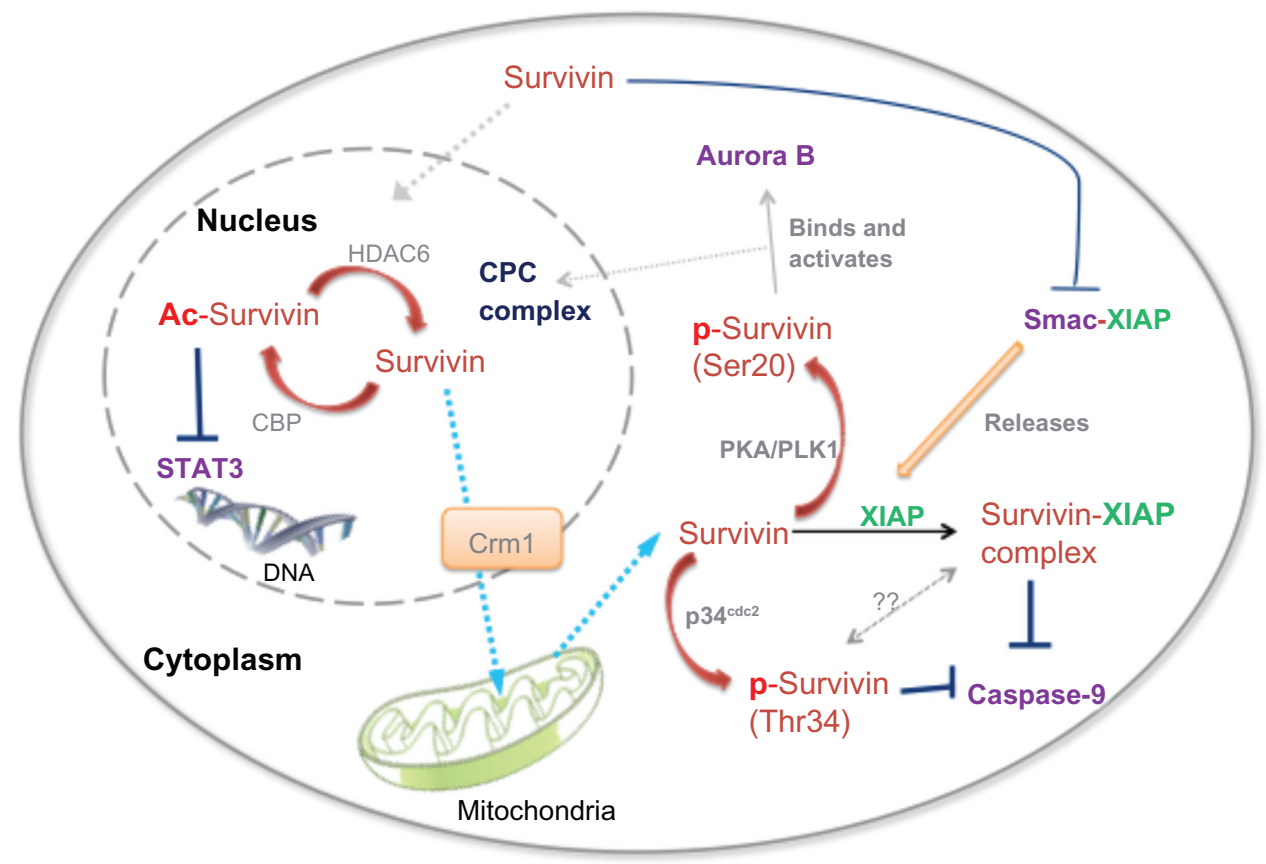

Figure 2 Post-translational regulations and functions of survivin.

Abbreviations: CPC, chromosomal passenger complex; STAT3, signal transducer and activator of transcription 3; Crm I, chromosome region maintenance I; PKA, protein kinase A; PLKI, polo-like kinase I; Smac, second mitochondria-derived activator of caspases; XIAP, X-linked inhibitor of apoptosis protein; HDAC6, histone deacetylase 6; Thr, threonine; Ser, serine; CBP, CREB-binding protein; Ac-Survivin, acetylated-Survivin; p-Survivin, phosphorylated-Survivin. 
of survivin in both apoptosis and mitosis (Figure 2). It is also worth noting that Aurora B could in return phosphorylate survivin on Thr117 in CPC. ${ }^{45,46}$ Delacour-Larose et $\mathrm{al}^{46}$ have demonstrated that the survivin phosphorylation dead mutant, T117A, could associate with centromeres and induce prometaphasic arrest in a survivin-depleted cell.

\section{Acetylation of survivin}

As we have discussed in the previous sections, survivin is present in both the mitochondria and the cytosol. In fact, survivin can also shuttle between the nucleus and the cytoplasm through active nucleocytoplasmic transport. Chromosome region maintenance 1 (Crm1/Xpo1) is a nuclear export receptor. It can interact with proteins containing the leucine-rich nuclear export signals and facilitates their nuclear export. ${ }^{47} \mathrm{Crm} 1$ is also a critical RAs-related nuclear protein (Ran-GTP) effector for mitotic spindle assembly and function in cells. ${ }^{48}$ Early studies revealed that survivin contains a nuclear export signal (possibly sequences: ${ }^{89}$ VKKQFEELTL ${ }^{98}$ or ${ }^{96}$ LTLGEFLKL $^{104}$ ) located between the BIR domain and the $\mathrm{COOH}$-terminal $\alpha$-helical coil, and it is exported to the cytoplasm through an interaction with Crm $1 .{ }^{49,50}$ In addition, abrogation of the cytoplasmic translocation of survivin has been shown to promote caspasedependent apoptosis in cancer cells. It was also shown that: (1) survivin dimerization occurs when leucine (Leu)98 of one of the molecules protrudes into a hydrophobic pocket, formed with Leu6, tryptophan (Trp)10, phenylalanine (Phe)93, Phe101, and Leu102 of the other molecule; and (2) homodimerization of survivin decreases its binding affinity to $\mathrm{Crm} 1$ and inhibits the nuclear export of survivin. ${ }^{42,51}$ However, factors affecting the transition between monomer and dimer of survivin were unclear. Interestingly, recent studies reveal that cAMP response element-binding protein (CREB)-binding protein (CBP) acetylates survivin on lysine 129 position to promote survivin homodimerization and the subsequent nuclear accumulation (Figure 2). These studies also reveal that cytoplasmic survivin inhibits apoptosis, whereas nuclear survivin may inhibit cell survival. ${ }^{52}$ It is not surprising to see that survivin could inhibit apoptosis in the cytoplasm because caspases such as caspase- 9 and caspase- 3 are mainly present in the cytoplasm. However, it is surprising to find that nuclear survivin could inhibit the transcription of various pro-survival genes in cancer cells. The Wang et $\mathrm{al}^{52}$ study reveals that CBP promotes survivin acetylation in the nucleus. In addition, the acetylated form of survivin binds to the N-terminal of signal transducer and activator of transcription 3 (STAT3) and represses its oncogenic activity. In contrast, deacetylation of survivin inhibits its homodimerization and promotes heterodimerization of survivin with Crm1 with subsequent nuclear export. ${ }^{52}$ The same research group also found that histone deacetylase 6 (HDAC6) plays an important role in survivin deacetylation and the subsequent nuclear export of survivin (Figure 2). CBP-dependent nuclear translocation of HDAC6 was shown in MCF7 breast cancer cells following estrogen treatment to regulate the acetylation state of survivin in the nucleus. ${ }^{53}$ It is widely accepted that nuclear survivin has reduced stability as compared with the cytoplasmic survivin, ${ }^{54,55}$ and that survivin promotes mitosis through formation of the CPC complex (together with INCENP and aurora B kinase) in the nucleus. In contrast, cytoplasmic survivin inhibits apoptosis through direct/indirect inhibition of caspases. ${ }^{54,55}$ Therefore, it will be important to determine whether the amount of survivin present in the nucleus is enough to induce a pro-apoptotic effect through STAT3 inhibition in cells in the future.

\section{Possible mitosis- and apoptosis- unrelated functions of survivin}

Growing evidence indicates that survivin interferes with the process of cell autophagy and down-regulation of survivin may induce apoptosis through autophagy-dependent mechanisms. ${ }^{56,57}$ An interaction between the autophagy regulator beclin-1 and survivin has been shown in human glioma cells in response to tumor necrosis factor-related apoptosisinducing ligand (TRAIL). ${ }^{58}$ Interestingly, a recent study shows that XIAP inhibits autophagy via the E3 ubiquitin-protein ligase (Mdm2)-p53 signaling pathway. Thus, survivin may also indirectly inhibit autophagy through interactions with XIAP and interference with the Mdm2-p53 signaling pathway. ${ }^{59}$ Besides mitosis, apoptosis and autophagy, a recent report shows that the translocation of survivin into the nucleus may enhance DNA double strand breaks (DBD) repair capability in cancer cells by up-regulating the molecular sensor of DNA damage, $\mathrm{Ku} 70{ }^{60,61}$ However, the mechanism/s in which survivin regulates autophagy and DNA repair remains to be determined.

\section{The potential of survivin as a therapeutic/drug target}

"Druggable" proteins do not necessarily become therapeutic targets, and vice versa, promising therapeutic targets do not necessarily become druggable proteins. Although survivin is an important oncogenic molecule widely expressed in various types of tumor and its overexpression is positively correlated with the progression and metastasis of tumor in patients; survivin was only considered as a "semi-druggable" target in the past because: (1) survivin does not contain a kinase domain (ie, not a protein kinase); and (2) survivin exhibits multiple functions. Therefore, it is difficult to use any high-throughput 
assay (eg, in vitro kinase assay) or molecular assay (eg, Western blot analysis) to screen potential survivin inhibitors.

However, improved understanding of the biology of survivin does create opportunities for scientists to develop different survivin-targeted therapies with differential cellular or molecular functions. For example, delivering phosphorylation-dead mutation survivin, T34A, into cancer cells may be a potential cancer therapeutic strategy. In theory, the T34A survivin mutant should be capable of forming a heterodimer with the wild-type survivin, and subsequently reducing the interaction between survivin and caspase-9. In fact, several studies have revealed the anti-cancer effect of delivering T34A plasmid DNA or recombinant protein by different nanocarrier approaches. Zhang et $\mathrm{al}^{62}$ successfully transported mouse-survivin-T34A plasmid DNA by utilizing heparin-polyethyleneimine (HPEI) nanoparticles. SurvivinT34A-loaded HPEI was shown to be capable of reducing colon cancer growth in vitro and in vivo. ${ }^{62}$ In addition, T34A mutant survivin could also enhance the chemotherapeutic sensitivity of drugs, including cisplatin and doxorubicin, in lung cancer cells and breast cancer cells. ${ }^{63,64}$ Besides the T34A mutant, a dominant-negative survivin (Cys84 $\rightarrow$ Ala, $\mathrm{C} 84 \mathrm{~A})$ protein fused to the cell-penetrating poly-arginine (R9) peptide has also been synthesized and was shown to be capable of inducing apoptosis in cancer cells. ${ }^{65}$ Interestingly, scientists have demonstrated that transduction of double point mutant (T34A and C84A) of survivin by adenovirus induced anti-cancer growth effect in hepatocellular cancer cells ${ }^{66}$ In fact, survivin T34A/C84A mutant (with double mutations) was shown to exhibit a stronger cancer killing effect as compared with the T34A or C84A survivin mutant (single mutation). Survivin contains a single BIR domain, and it is believed that the BIR domain plays an important role in physically interacting between survivin and second mitochondria-derived activator of caspases (Smac)/DIABLO ${ }^{67} \mathrm{Smac} / \mathrm{DIABLO}$ is a mitochondrial protein that is released upon apoptotic stimulation by ultraviolet irradiation and ionizing irradiation. It has also been shown that Smac/ DIABLO binds to XIAP. ${ }^{68}$ Researchers suggest that Smac/ DIABLO and caspase- 9 bind to overlapping sites on the BIR3 domain of XIAP. They also suggest that Smac/DIABLO acts by releasing caspase-9 from the XIAP/caspase- 9 complex, leading to apoptosis activation. In contrast, survivin binds to Smac/DIABLO and inhibits its pro-apoptotic functions through releasing of XIAP from the Smac/DIABLO/XIAP complex to inhibit various caspases. In theory, C84A mutation in the extreme C-terminal region of the BIR domain can disrupt the $\mathrm{Zn}^{2+}$ coordination sphere and subsequently inhibit the interactions between survivin and Smac/DIABLO. Therefore, T34A/C84A survivin mutant may induce cancer cell death through two distinct mechanisms: (1) by forming a heterodimer with the wild-type survivin, leading to the reduced interaction with caspase-9; and (2) by reducing the interaction between survivin and Smac/DIABLO, leading to an increased binding between Smac/DIABLO and XIAP, resulting in the activity inhibition of XIAP.

Besides T34A and C84A survivin mutants, Song et $\mathrm{al}^{69}$ showed a novel survivin mutant, Asp53 $\rightarrow$ Ala (D53A), which had a function similar as T34A; several cellular functions of D53A were demonstrated. Asp53 is located within the BIR domain of survivin; it induced $\mathrm{p} 53$-independent apoptosis by forming a heterodimer with wild type survivin or forming

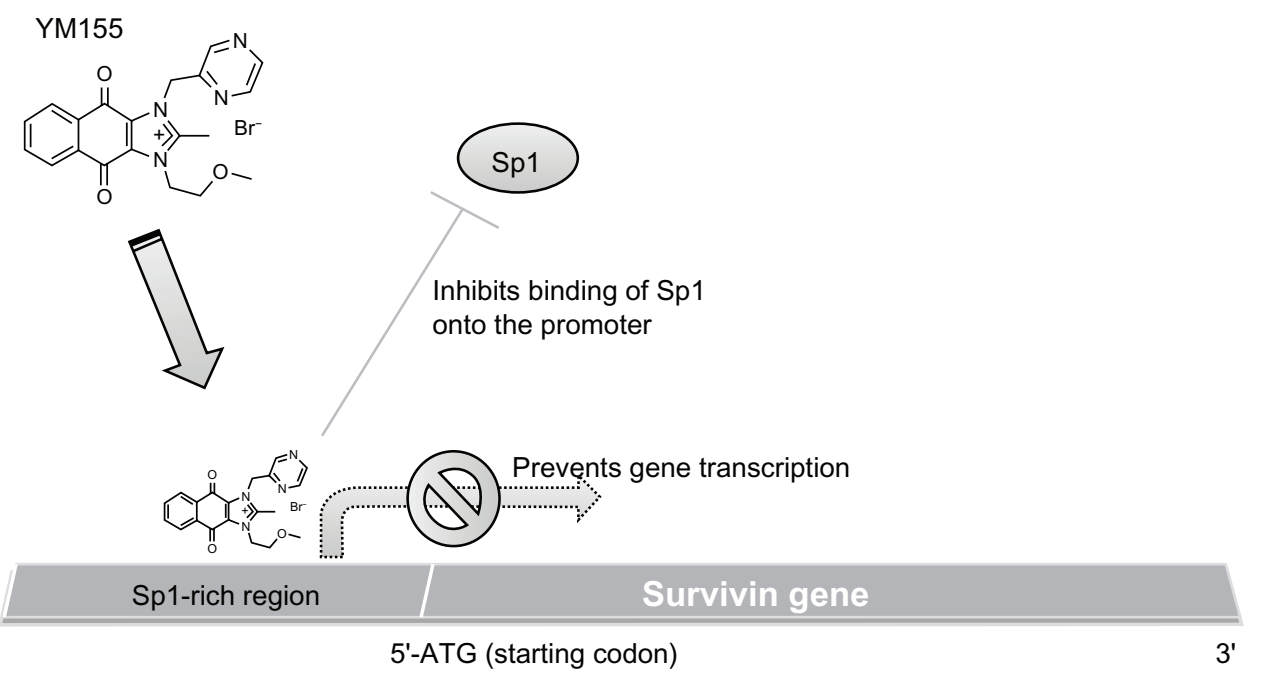

Figure 3 Chemical structure and functions of the survivin small molecule inhibitor, YMI55. YMI 55 binds onto the specificity protein I (Sp I)-rich region of survivin promoter and inhibits survivin gene transcription. 


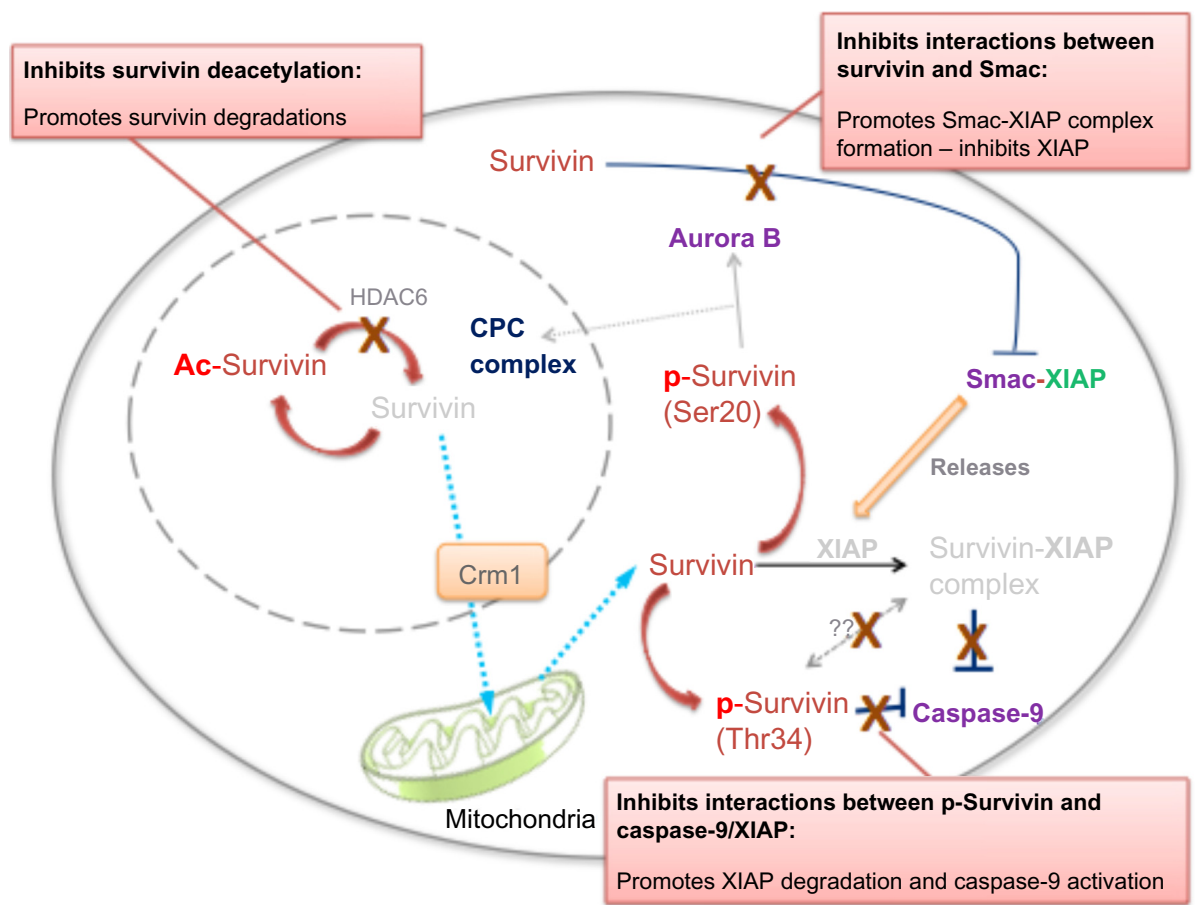

Figure 4 Future strategies of targeting survivin. Peptides or small molecule inhibitors can be developed to interfere with the interactions between survivin and its binding partners to either promote survivin degradation or to release the inhibition of various pro-apoptotic molecules.

Abbreviations: CPC, chromosomal passenger complex; STAT3, signal transducer and activator of transcription 3; Crm I, chromosome region maintenance I; Smac, second mitochondria-derived activator of caspases; XIAP, X-linked inhibitor of apoptosis protein.

a homodimer to abolish anti-apoptotic activity of wild type survivin. In addition, the formation of both the homo- and heterodimer abolished binding to Smac/DIABLO. ${ }^{69}$

\section{Conclusion}

Survivin plays multiple roles in the promotion of cancer cell survival. Surprisingly, only a few small molecule inhibitors were developed in the past, and only one of them, YM155 (survivin gene transcription inhibitor), has reached Phase II clinical trial (Figure 3). Although the development of survivin small molecule inhibitors has been limited in the past, recent advances in survivin biology do create opportunities for scientists to target survivin with various function-specific dominant-negative proteins/ peptides/small molecule inhibitors that are capable of abolishing the interactions between survivin and its differential binding partners (Figure 4). Recent advances in nanotechnology also increase the possibility for using dominant-negative proteins, peptides or small interfering RNA as therapeutic agents in targeting survivin-positive cancer cells, given that application of nanoparticle encapsulation may enhance the stability of macromolecular drugs and further increase their functionality in vivo and in the human body. ${ }^{70}$ Taken together, these scientific and technical advances open up new possibility for therapeutic strategies that can be applied to target a specific function of survivin that is crucial for cancer cell survival, yet preserve functions of survivin that are important for normal cell survival.

\section{Acknowledgments}

This work is kindly supported by the following grants: NSC101-2320-B-006-041 and NSC102-2320-B-006-038 (National Science Council, Taiwan, Republic of China); NHRI-EX102-10237SC (National Health Research Institutes, Taiwan, Republic of China); "Aim for the Top University Project for National Cheng Kung University" (Ministry of Education, Taiwan, Republic of China); and CMNCKU10111 (Chi Mei Medical Center, Taiwan, Republic of China).

\section{Disclosure}

The authors declare that they have no competing interests.

\section{References}

1. Waligórska-Stachura J, Jankowska A, Waśko R, et al. Survivin prognostic tumor biomarker in human neoplasms - review. Ginekol Pol. 2012;83(7):537-540.

2. Farnebo L, Tiefenböck K, Ansell A, Thunell LK, Garvin S, Roberg K. Strong expression of survivin is associated with positive response to radiotherapy and improved overall survival in head and neck squamous cell carcinoma patients. Int J Cancer. 2013;133(8):1994-2003. 
3. Lu CD, Altieri DC, Tanigawa N. Expression of a novel antiapoptosis gene, survivin, correlated with tumor cell apoptosis and p53 accumulation in gastric carcinomas. Cancer Res. 1998;58(9):1808-1812.

4. Adida C, Haioun C, Gaulard P, et al. Prognostic significance of survivin expression in diffuse large B-cell lymphomas. Blood. 2000;96(5): 1921-1925.

5. Gu X, Lin HL. Analysis of survivin expression in subtypes of lymphoma. Ai Zheng. 2004;23(6):655-661. Chinese.

6. Sarela AI, Macadam RC, Farmery SM, Markham AF, Guillou PJ. Expression of the antiapoptosis gene, survivin, predicts death from recurrent colorectal carcinoma. Gut. 2000;46(5):645-650.

7. Kawasaki H, Toyoda M, Shinohara H, et al. Expression of survivin correlates with apoptosis, proliferation, and angiogenesis during human colorectal tumorigenesis. Cancer. 2001;91(11):2026-2032.

8. Hsu KF, Lin CK, Yu CP, et al. Cortactin, fascin, and survivin expression associated with clinicopathological parameters in esophageal squamous cell carcinoma. Dis Esophagus. 2009;22(5):402-408.

9. Chantalat L, Skoufias DA, Kleman JP, Jung B, Dideberg O, Margolis RL. Crystal structure of human survivin reveals a bow tie-shaped dimer with two unusual alpha-helical extensions. Mol Cell. 2000;6(1): 183-189.

10. Coumar MS, Tsai F-Y, Kanwar JR, Sarvagalla S, Cheung CHA. Treat cancers by targeting survivin: Just a dream or future reality? Cancer Treat Rev. 2013;39(7):802-811.

11. Kawamura K, Yu L, Tomizawa M, et al. Transcriptional regulatory regions of the survivin gene activate an exogenous suicide gene in human tumors and enhance the sensitivity to a prodrug. Anticancer Res. 2007;27(1A):89-93.

12. Li FZ, Altieri DC. Transcriptional analysis of human survivin gene expression. Biochemical Journal. 1999;344:305-311.

13. Xu R, Zhang P, Huang J, Ge S, Lu J, Qian G. Sp1 and Sp3 regulate basal transcription of the survivin gene. Biochem Biophys Res Commun. 2007;356(1):286-292.

14. Chen Y, Wang X, Li W, et al. Sp1 upregulates survivin expression in adenocarcinoma of lung cell line A549. Anat Rec (Hoboken). 2011;294(5):774-780.

15. Jiang Y, Saavedra HI, Holloway MP, Leone G, Altura RA. Aberrant regulation of survivin by the $\mathrm{RB} / \mathrm{E} 2 \mathrm{~F}$ family of proteins. J Biol Chem. 2004;279(39):40511-40520.

16. Hoffman WH, Biade S, Zilfou JT, Chen J, Murphy M. Transcriptional repression of the anti-apoptotic survivin gene by wild type p53. J Biol Chem. 2002;277(5):3247-3257.

17. Meng RD, Shelton CC, Li YM, et al. gamma-Secretase inhibitors abrogate oxaliplatin-induced activation of the Notch-1 signaling pathway in colon cancer cells resulting in enhanced chemosensitivity. Cancer Res. 2009;69(2):573-582.

18. Chen Y, Li D, Liu H, et al. Notch-1 signaling facilitates survivin expression in human non-small cell lung cancer cells. Cancer Biology and Therapy. 2011;11(1):14-21.

19. Vaira V, Lee CW, Goel HL, Bosari S, Languino LR, Altieri DC. Regulation of survivin expression by IGF-1/mTOR signaling. Oncogene. 2007;26(19):2678-2684.

20. Kimball SR, Jefferson LS. Signaling pathways and molecular mechanisms through which branched-chain amino acids mediate translational control of protein synthesis. J Nutr. 2006;136(Suppl 1):227S-231S.

21. Li F, Ambrosini G, Chu EY, et al. Control of apoptosis and mitotic spindle checkpoint by survivin. Nature. 1998;396(6711):580-584.

22. Zhao J, Tenev T, Martins LM, Downward J, Lemoine NR. The ubiquitinproteasome pathway regulates survivin degradation in a cell cycledependent manner. J Cell Sci. 2000;113 Pt 23:4363-4371.

23. Young JC, Moarefi I, Hartl FU. Hsp90: a specialized but essential protein-folding tool. J Cell Biol. 2001;154(2):267-273.

24. Fortugno P, Beltrami E, Plescia J, et al. Regulation of survivin function by Hsp90. Proc Natl Acad Sci U S A. 2003;100(24):13791-13796.

25. Wang $\mathrm{H}$, Gambosova $\mathrm{K}$, Cooper ZA, et al. EGF regulates survivin stability through the Raf-1/ERK pathway in insulin-secreting pancreatic beta-cells. BMC Mol Biol. 2010;11:66.
26. Ju JH, Yang W, Oh S, et al. HER2 stabilizes survivin while concomitantly down-regulating survivin gene transcription by suppressing Notch cleavage. Biochem J. 2013;451(1):123-134.

27. Siddiqa A, Long LM, Li L, Marciniak RA, Kazhdan I. Expression of HER-2 in MCF-7 breast cancer cells modulates anti-apoptotic proteins Survivin and Bcl-2 via the extracellular signal-related kinase (ERK) and phosphoinositide-3 kinase (PI3K) signalling pathways. BMC Cancer. 2008;8:129.

28. Wall NR, O'Connor DS, Plescia J, Pommier Y, Altieri DC. Suppression of survivin phosphorylation on Thr34 by flavopiridol enhances tumor cell apoptosis. Cancer Res. 2003;63(1):230-235.

29. Beierle EA, Nagaram A, Dai W, Iyengar M, Chen MK. VEGF-mediated survivin expression in neuroblastoma cells. J Surg Res. 2005;127(1): 21-28.

30. Blanc-Brude OP, Yu J, Simosa H, Conte MS, Sessa WC, Altieri DC. Inhibitor of apoptosis protein survivin regulates vascular injury. Nat Med. 2002;8(9):987-994.

31. Yu J, Zhang YY, Zhang XB, et al. Endothelium derived nitric oxide synthase negatively regulates the PDGF-survivin pathway during flow-dependent vascular remodeling. Plos One. 2012;7(2):e31495

32. Xu Y, Fang F, Ludewig G, Jones G, Jones D. A mutation found in the promoter region of the human Survivin gene is correlated to overexpression of survivin in cancer cells. DNA Cell Biology. 2004;23(9): 527-537.

33. Tsuji N, Furuse K, Asanuma K, et al. Mutations of the p53 gene and loss of heterozygosity at chromosome $17 \mathrm{p} 13.1$ are associated with increased survivin expression in breast cancer. Breast Cancer Res Treat. 2004;87(1):23-31.

34. Li Y, Xie M, Yang J, et al. The expression of antiapoptotic protein survivin is transcriptionally upregulated by DEC1 primarily through multiple sp1 binding sites in the proximal promoter. Oncogene. 2006;25(23):3296-3306.

35. O'Connor DS, Grossman D, Plescia J, et al. Regulation of apoptosis at cell division by $\mathrm{p} 34 \mathrm{cdc} 2$ phosphorylation of survivin. Proc Natl Acad Sci U S A. 2000;97(24):13103-13107.

36. Zhou S, Li L, Jian X, et al. The phosphorylation of survivin Thr34 by p34cdc 2 in carcinogenesis of oral submucous fibrosis. Oncol Rep. 2008;20(5):1085-1091.

37. Pannone G, Bufo P, Serpico R, et al. Survivin phosphorylation and M-phase promoting factor in oral carcinogenesis. Histol Histopathol. 2007;22(11):1241-1249.

38. Barrett RM, Osborne TP, Wheatley SP. Phosphorylation of survivin at threonine 34 inhibits its mitotic function and enhances its cytoprotective activity. Cell Cycle. 2009;8(2):278-283.

39. Dohi T, Xia F, Altieri DC. Compartmentalized phosphorylation of IAP by protein kinase A regulates cytoprotection. Mol Cell. 2007;27(1): 17-28

40. Chu Y, Yao PY, Wang W, et al. Aurora B kinase activation requires survivin priming phosphorylation by PLK1. J Mol Cell Biol. 2011;3(4): 260-267.

41. Dohi T, Beltrami E, Wall NR, Plescia J, Altieri DC. Mitochondrial survivin inhibits apoptosis and promotes tumorigenesis. J Clin Invest. 2004;114(8):1117-1127.

42. Jeyaprakash AA, Klein UR, Lindner D, et al. Structure of a Survivin-Borealin-INCENP core complex reveals how chromosomal passengers travel together. Cell. 2007;131(2):271-285.

43. Bolton MA, Lan WJ, Powers SE, McCleland ML, Kuang J, Stukenberg PT. Aurora B kinase exists in a complex with survivin and INCENP and its kinase activity is stimulated by survivin binding and in a complex inase activity is phosphorylation. Mol Biol Cell. 2002;13(9): 3064-3077.

44. Colnaghi R, Wheatley SP. Liaisons between survivin and Plk1 during cell division and cell death. J Biol Chem. 2010;285(29):22592-22604.

45. Wheatley SP, Henzing AJ, Dodson H, Khaled W, Earnshaw WC. Aurora-B phosphorylation in vitro identifies a residue of survivin that is essential for its localization and binding to inner centromere protein (INCENP) in vivo. J Biol Chem. 2004;279(7):5655-5660. 
46. Delacour-Larose M, Thi MN, Dimitrov S, Molla A. Role of survivin phosphorylation by aurora B in mitosis. Cell Cycle. 2007;6(15): 1878-1885.

47. Hutten S, Kehlenbach RH. CRM1-mediated nuclear export: to the pore and beyond. Trends Cell Biol. 2007;17(4):193-201.

48. Arnaoutov A, Azuma Y, Ribbeck K, et al. Crm1 is a mitotic effector of Ran-GTP in somatic cells. Nat Cell Biol. 2005;7(6):626-632.

49. Knauer SK, Bier C, Habtemichael N, Stauber RH. The SurvivinCrm1 interaction is essential for chromosomal passenger complex localization and function. EMBO Rep. 2006;7(12):1259-1265.

50. Knauer SK, Kramer OH, Knosel T, et al. Nuclear export is essential for the tumor-promoting activity of survivin. FASEB J. 2007;21(1): 207-216.

51. Engelsma D, Rodriguez JA, Fish A, Giaccone G, Fornerod M. Homodimerization antagonizes nuclear export of survivin. Traffic. 2007;8(11):1495-1502.

52. Wang H, Holloway MP, Ma L, et al. Acetylation directs survivin nuclear localization to repress STAT3 oncogenic activity. J Biol Chem. 2010;285(46):36129-36137.

53. Riolo MT, Cooper ZA, Holloway MP, et al. Histone deacetylase 6 (HDAC6) deacetylates survivin for its nuclear export in breast cancer. J Biol Chem. 2012;287(14):10885-10893.

54. Connell CM, Colnaghi R, Wheatley SP. Nuclear survivin has reduced stability and is not cytoprotective. J Biol Chem. 2008;283(6): 3289-3296.

55. Chan KS, Wong CH, Huang YF, Li HY. Survivin withdrawal by nuclear export failure as a physiological switch to commit cells to apoptosis. Cell Death Dis. 2010;1:e57.

56. Roca H, Varsos Z, Pienta KJ. CCL2 protects prostate cancer PC3 cells from autophagic death via phosphatidylinositol 3-kinase/AKT-dependent survivin up-regulation. J Biol Chem. 2008;283(36):25057-25073.

57. Wang QW, Chen ZT, Diao XW, Huang SB. Induction of autophagydependent apoptosis by the survivin suppressant YM155 in prostate cancer cells. Cancer Lett. 2011;302(1):29-36.

58. Niu TK, Cheng Y, Ren XC, Yang JM. Interaction of Beclin 1 with survivin regulates sensitivity of human glioma cells to TRAIL-induced apoptosis. FEBS Lett. 2010;584(16):3519-3524.
59. Huang $X, W u Z$, Mei Y, Wu M. XIAP inhibits autophagy via XIAP-Mdm2-p53 signalling. EMBO J. 2013;32(16):2204-2216.

60. Jiang GF, Ren B, Xu L, Song S, Zhu C, Ye F. Survivin may enhance DNA double-strand break repair capability by up-regulating Ku70 in human KB cells. Anticancer Res. 2009;29(1):223-228.

61. Capalbo G, Dittmann K, Weiss C, et al. Radiation-Induced Survivin Nuclear Accumulation Is Linked to DNA Damage Repair. Int J Radiat Oncol Biol Phys. 2010;77(1):226-234.

62. Zhang L, Gao X, Men K, et al. Gene therapy for C-26 colon cancer using heparin-polyethyleneimine nanoparticle-mediated survivin T34A. Int J Nanomedicine. 2011;6:2419-2427.

63. Yu DD, Wang CT, Shi HS, et al. Enhancement of cisplatin sensitivity in Lewis Lung carcinoma by liposome-mediated delivery of a survivin mutant. J Exp Clin Cancer Res. 2010;29:46.

64. Xu Y, Zheng W, Wang T, Wang P, Zhu L, Ma X. Genetic protein TmSm(T34A) enhances sensitivity of chemotherapy to breast cancer cell lines as a synergistic drug to doxorubicin. Biomed Pharmacother. 2012;66(5):368-372.

65. Cheung CHA, Sun XY, Kanwar JR, Bai JZ, Cheng L, Krissansen GW. A cell-permeable dominant-negative survivin protein induces apoptosis and sensitizes prostate cancer cells to TNF-alpha therapy. Cancer Cell Int. 2010;10:36

66. Zhang R, Wang T, Li KN, et al. A survivin double point mutant has potent inhibitory effect on the growth of hepatocellular cancer cells. Cancer Biol Ther. 2008;7(4):547-554.

67. Song ZY, Yao XB, Wu M. Direct interaction between survivin and Smac/ DIABLO is essential for the anti-apoptotic activity of survivin during taxol-induced apoptosis. J Biol Chem. 2003;278(25):23130-23140.

68. Liu ZH, Sun CH, Olejniczak ET, et al. Structural basis for binding of Smac/DIABLO to the XIAP BIR3 domain. Nature. 2000;408(6815): 1004-1008.

69. Song ZY, Liu SX, He H, et al. A single amino acid change (Asp 53 -> Ala53) converts survivin from anti-apoptotic to pro-apoptotic. Mol Biol Cell. 2004;15(3):1287-1296.

70. Becker AL, Orlotti NI, Folini M, et al. Redox-active polymer microcapsules for the delivery of a survivin-specific siRNA in prostate cancer cells. Acs Nano. 2011;5(2):1335-1344.
OncoTargets and Therapy

\section{Publish your work in this journal}

OncoTargets and Therapy is an international, peer-reviewed, open access journal focusing on the pathological basis of all cancers, potential targets for therapy and treatment protocols employed to improve the management of cancer patients. The journal also focuses on the impact of management programs and new therapeutic agents and protocols on

\section{Dovepress}

patient perspectives such as quality of life, adherence and satisfaction The manuscript management system is completely online and includes a very quick and fair peer-review system, which is all easy to use. Visit http://www.dovepress.com/testimonials.php to read real quotes from published authors. 